\title{
The Janus face of rosiglitazone
}

\section{Christine Pich and Liliane Michalik}

\author{
Thiazolidinediones: anti-diabetic drugs to treat \\ cancer?
}

Thiazolidinediones (TZDs) are potent insulin sensitizers used to prevent and treat type 2 diabetes [1]. Widely-used in the early 2000', pharmacological treatments with these compounds have been drastically reduced due to adverse effects, like weight gain, hepatotoxicity, and congestive heart failure $[1,2]$. Nevertheless, two members of this family of compounds, rosiglitazone (Avandia) and pioglitazone (Actos), are still approved for the treatment of type 2 diabetes in selected patients and under restricted conditions.

Besides treatment of diabetes, TZDs have been proposed as a therapeutic option for cancer. This was initially based on the observation that the nuclear receptor PPAR $\gamma$-the best characterized molecular target of TZDsexhibits anti-cancer properties. PPAR $\gamma$ is involved in the regulation of several conditions that are key for controlling tumor growth, like cell proliferation, cell survival, cell differentiation, and inflammation $[2,3]$. Moreover, genetic evidence shows that loss of PPAR $\gamma$ function predisposes to colon, breast, ovary and skin cancers [4]. Finally numerous studies have reported anti- tumoral effects of PPAR $\gamma$ activation both in vitro and in in vivo mouse models, possibly also in humans [4]. Despite anti-cancer properties reported in many cancer cell types, clinical trials involving TZDs and activation of PPAR $\gamma$, which were performed over the past 15 years have been disappointing, showing little therapeutic efficacy [2].

Undesirable pro-tumorigenic actions of rosiglitazone in metastatic human melanoma cells.

Skin melanoma, an aggressive malignant neoplasm of melanocytes, is the most dangerous form of skin cancer. Although it represents only $4 \%$ of all skin cancers, it is currently responsible for $80 \%$ of skin cancer-related deaths. Cancer research has taught us that melanoma require multiple therapeutic approaches. The search for treatments against melanoma is therefore still active, despite the recent breakthrough of immuno- and molecularly targeted therapies [5].

Several studies conducted over the past 20 years supported the use of TZDs and of PPAR $\gamma$ activation as a therapeutic option to treat melanoma [6]. However, these studies showed anti-cancer effects on the malignant cells (Figure 1), while they neglected the role played by nonmalignant cells of a tumor microenvironment. Recently,

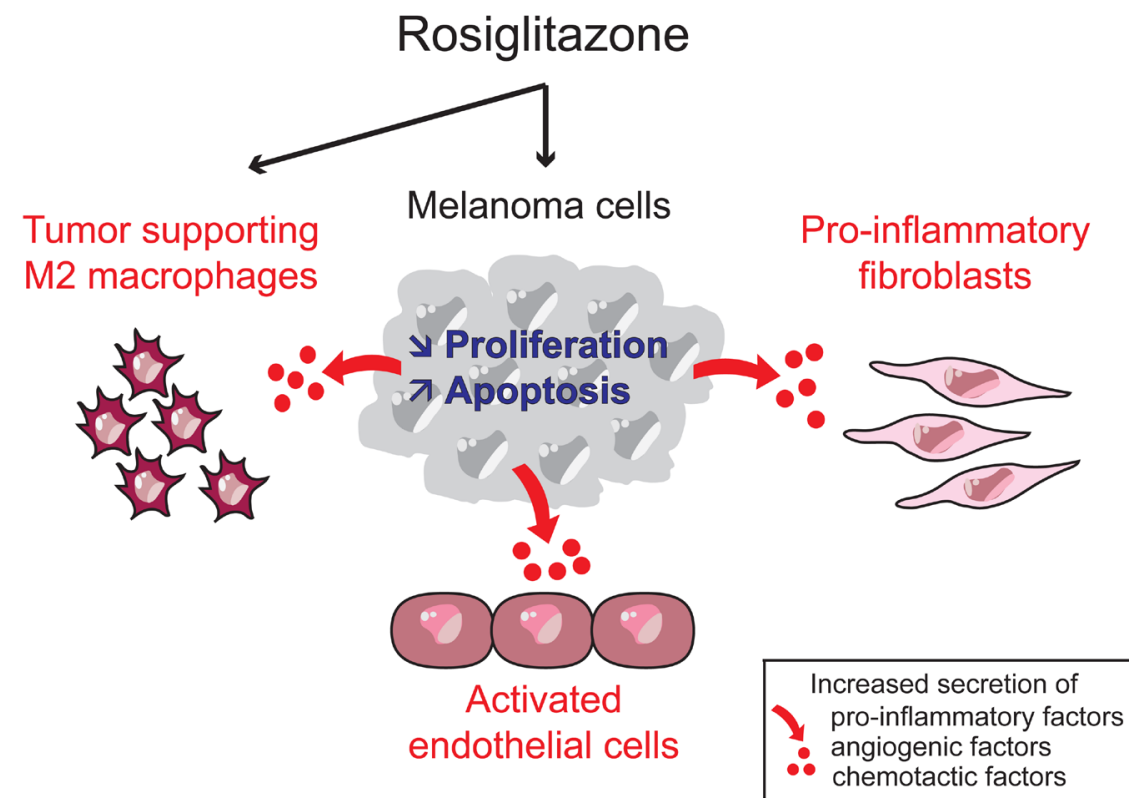

Figure 1: The Janus face of rosiglitazone in melanoma. On the one hand, rosiglitazone shows cell-autonomous anti-cancer actions (in blue) in melanoma cells, by preventing proliferation and promoting apoptosis [6]. On the other hand, rosiglitazone shows pro-cancer actions (in red). These include cell-autonomous impact on macrophages [6], as well as paracrine effects on fibroblasts, endothelial cells and tumor associated macrophages [7]. Of note, while the metabolic actions of rosiglitazone are clearly mediated by PPAR $\gamma$, its actions on cancer cells can only partially be explained by PPAR $\gamma$ activation [2]. The figure was inspired by discussion with Dr Thanh Nhan N'Guyen. 
a study of TZDs impacts extended beyond the melanoma cell itself, and identified a new pro-tumorigenic paracrine action of rosiglitazone (RGZ) [7]. This pro-tumorigenic action of RGZ involves increased secretion of cytokines, chemokines and angiogenic factors by a subset of human melanoma cells, in turn activating non-malignant fibroblasts, endothelial cells and macrophages in a tumorfriendly way (Figure 1). In mice, tumors derived from human melanoma cells and exposed to RZG exhibited accelerated development, and increased inflammation and angiogenesis. In this model of melanoma, RGZ pro-tumorigenic action prevailed over its anti-cancer action [7]. In addition, in line with these results, RGZ retained this pro-tumorigenic effect on human melanoma cells exposed to Vemurafenib, a recently approved pharmacological treatment of melanoma targeting the BRAF mutant $\mathrm{BRAF}^{\mathrm{V} 600 \mathrm{E}}$ [8] [Pich et al, unpublished results]. In these melanoma cells, RGZ partially prevented Vemurafenib from inhibiting proliferation and interleukins secretion, thereby reducing Vemurafenib efficacy.

Collectively, these data suggest that adverse consequences may result from exposing patients with existing melanoma to RGZ, and potentially to other PPAR $\gamma$ agonists. Finally, although focusing on melanoma, the study by Pich and collaborators suggests that RGZ may have similar undesirable impacts in other pre-existing tumors, like non-melanoma skin cancers and bladder cancers [7].

Thiazolidinediones as an option to treat cancer: balancing pro-tumorigenic and anti-cancer effects.

The vast majority of studies conducted on cohorts of diabetic patients with no pre-existing tumors report overall preventive benefits of TZDs, with the exception of pioglitazone, which was suspected to slightly increase the risk of bladder cancers, non-Hodgkin lymphoma and melanoma [2,9]. For existing tumors, investigation of TZDs as a therapeutic option led to contradictory findings, showing pro-tumorigenic effects on the one hand and anti-cancer effects on the other hand. These contrasting results seem to result from a combination of complex PPAR $\gamma$-dependent or -independent impacts, contextand compound- specific actions and, as shown in [7], pro-tumorigenic effects on the tumor microenvironment opposing the anti-cancer effects observed on the malignant cells (Figure 1). Recent progress in omics and single cell techniques will likely allow for a better understanding of how the various cells types in a tumor respond to TZDs.

TZDs complex and contrasting actions make it impossible to generalize the impact of pharmacological treatment in regard to cancer development. Moreover, they partially explain why promising in vitro studies have not been translated into convincing clinical trials. Besides TZDs, the search for novel PPAR $\gamma$ agonists is still active [10] and their impact on cancer cells is still questioned.
Finding PPAR $\gamma$ agonists that retain anti-cancer activities but lack pro-tumorigenic properties would be potential therapeutic options for cancer treatment.

Liliane Michalik: Center for Integrative Genomics, University of Lausanne, Lausanne, Switzerland

Correspondence to: Liliane Michalik, email liliane.michalik@unil.ch

Keywords: rosiglitazone; PPARY; melanoma; tumor microenvironment

Received: December 17, 2018

Published: December 28, 2018

\section{REFERENCES}

1. Soccio RE, et al. Cell Metab. 2014; 20: 573-91.

2. Frohlich E, et al. Biomed Res Int. 2015; 2015:845340.

3. Gou Q, et al. Oncotarget. 2017; 8:60704-9. https://doi.org/10.18632/oncotarget.19610

4. Robbins GT, et al. Front Biosci (Landmark Ed). 2012; 17:1816-34.

5. Michielin O, et al. Cancer Treat Rev. 2015; 41:660-70.

6. Gupta M, et al. Arch Dermatol Res. 2015; 307:767-80.

7. Pich C, et al. Cancer Res. 2018; 78:6447-61.

8. Flaherty KT,et al. N Engl J Med. 2010; 363: 809-19.

9. Ferrara A, et al. Diabetes Care. 2011; 34: 923-9.

10. Zhang J, et al. Chem Biol Drug Des. 2016; 88: 635-63.

Copyright: Pich et al. This is an open-access article distributed under the terms of the Creative Commons Attribution License 3.0 (CC BY 3.0), which permits unrestricted use, distribution, and reproduction in any medium, provided the original author and source are credited. 